\title{
Prototype WSN Platform for Performing Dynamic Monitoring of Civil Engineering Structures
}

\author{
Rafael Aguilar, Civil Engineering PhD student, Department of Civil Engineering - ISISE, University of \\ Minho, Campus de Azurém 4800 - 058 Guimarães, Portugal
}

Luís F. Ramos, PhD in Civil Engineering, Assistant Professor, Department of Civil Engineering ISISE, University of Minho, Campus de Azurém 4800 - 058 Guimarães, Portugal

Paulo B. Lourenço, PhD in Civil Engineering, Full Professor, Department of Civil Engineering - ISISE, University of Minho, Campus de Azurém 4800 - 058 Guimarães, Portugal

Ricardo Severino, Electrical and Computer Engineering PhD Student, CISTER/IPP-HURRAY! Research Unit - School of Engineering Polytechnic Institute of Porto, 4200-072 Porto, Portugal

Ricardo Gomes, Junior Researcher, CISTER/IPP-HURRAY! Research Unit - School of Engineering Polytechnic Institute of Porto, 4200-072 Porto, Portugal

Paulo Gandra, PhD in Electrical and Computer Engineering, Researcher, CISTER/IPP-HURRAY! Research Unit - School of Engineering Polytechnic Institute of Porto, 4200-072 Porto, Portugal

Mario Alves, PhD in Electrical and Computer Engineering, Researcher, CISTER/IPP-HURRAY! Research Unit - School of Engineering Polytechnic Institute of Porto, 4200-072 Porto, Portugal

Eduardo Tovar, PhD in Electrical and Computer Engineering, Research Leader, CISTER/IPPHURRAY! Research Unit - School of Engineering Polytechnic Institute of Porto, 4200-072 Porto, Portugal

\begin{abstract}
Structural Health Monitoring represents the present and future of the civil engineering since, until few years ago, structural diagnosis works had been performed with few resources regarding to experimental techniques. Precisely in the field of monitoring sensors, the progress of new technologies based on wireless communications and Micro-Electro-MechanicalSystems (MEMS) are of high interest for replacing the handle difficult wired sensors. However, three major limitations of the commercial off-the-shelf technology on WSN (combination of MEMS and wireless technology) for performing dynamic monitoring were identified by means of: (1) not enough sensitivity of the accelerometers; (2) low resolution of the ADC embedded; and (3) lack of synchronization algorithms implemented. This paper presents a new prototype system conceived for performing dynamic monitoring civil engineering structures. This system was jointly conceived by a team of civil, electrical and communication engineers and is a combination of the last technology on high resolution MEMS accelerometers and the state of the art of communication technologies. Despite the fact that the prototype system needs more improvements; the results of several rounds of validation experiences confirm the feasibility for its consideration as an alternative to the conventional wired based sensors.
\end{abstract}




\section{INTRODUCTION}

According to Farrar and Worden [10], dynamic wired based monitoring systems are being widely applied to study civil engineering structures since the early 1980s. Most of those studies are related to large structures by means of bridges and tall buildings. In the case of existent masonry structures, there are also several applications such as the study of temples [13], and masonry towers $[11 ; 12 ; 22 ; 23]$. These systems were also used to study other types of masonry structures like arch bridges $[8]$, churches $[4 ; 5 ; 20]$ and minarets $[21]$.

In the case of dynamic wireless based monitoring systems, the first case of study was the Alamosa Canyon Bridge in 1998 [24]. Afterwards, more case studies related with bridges were considered. These are the experiences in the Tokyo Rainbow Bridge in Japan [2], again the Alamosa Canyon Bridge in USA [15], a pedestrian bridge in the University of California-Irvine in USA [7], the Geumdang Bridge in Korea [16], the Gi-Lu cable-stayed bridge in Taiwan [25] and the Golden Gate Bridge in USA [18]. However, the only task reported in the literature related to large buildings is the dynamic monitoring of the 79 stories Di Wang Tower, located in Guangdong, China [17]. As masonry structures are difficult to excite and commercial MEMS have currently low resolution capabilities, only one case of dynamic wireless monitoring systems applications in existent masonry structures were reported. This study is the modal analysis of the Aquila Tower in Italy [6]. A novel contribution is given in this paper regarding the application of wireless based monitoring systems to other structures.

The paper starts by evaluating commercial off-the shelf WSN platforms in terms of its practical utility for performing structural dynamic monitoring works. Once the limitations of the available technology are stated, a new prototype system will be presented. Using this new prototype system, the results of several rounds of validations tests will be detailed. Finally, the conclusions as well as a draft of the future works are stated.

\section{OPERATIONAL MODAL ANALYSIS USING COMMERCIAL OFF-THE-SHELF WSN PLATFORMS}

Crossbow technology [9] was chosen as the best alternative for performing dynamic monitoring as it offers economical solutions including low powering boards and measurement platforms with microaccelerometers embedded. The selected Crossbow product (WSN professional kit) uses as gateway a Mica2 board in charge of the communication over the network in the radio frequency range of $868 / 916 \mathrm{MHz}$. To provide in-system programming and to supply energy to the devices, the system uses an interface board model MIB520 connected to a central computer trough an USB bus. The sensors' platform supplied by Crossbow is composed by a measurement board that works mounted on a Mica2 board. In this unit, the Mica2 board is not only in charge of the network communication but also in charge on supplying energy to the measurement board (using two AA batteries), as well as acquiring and converting the analogue measurements with an ADC resolution of 10 bits. There are several options of measurements boards available in the market, however; the one suitable for dynamic monitoring works is the MTS400. This measurement board has embedded four microsensors: biaxial microaccelerometer; light; pressure-temperature; and humidity-temperature. The location of the sensors in the MTS400 board and a view of the mounted measurement unit are shown in Figure 1. More details of the Data Acquisition (DAQ) process can be found at [1].

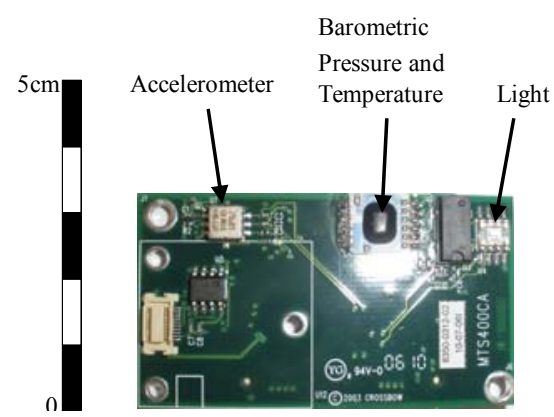

(a)

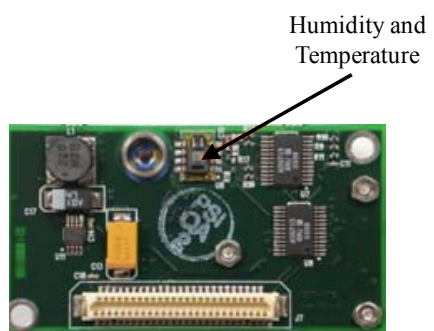

Figure 1 - Crossbow sensors' platform: (a) front and back of the MTS400 sensors' board; and (b) mounted unit

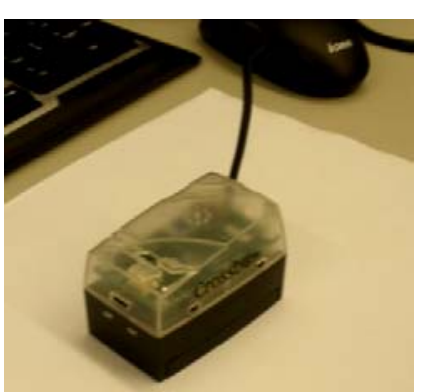

(b)

A single degree of freedom structure represented by an inverted pendulum is one of the simplest examples that are used by civil engineers to explain the fundamentals of the dynamics of structures. In this work, this structure is also used as a tool to evaluate and understand the behaviour of the commercial wireless based platforms and their utility for engineering works. For comparison purposes, conventional wired based systems composed by high sensitive accelerometers with a measurement range of $\pm 0.5 \mathrm{~g}$ and sensitivity of $10.000 \mathrm{mV} / \mathrm{g}$ as well as an ADC with 24 bits of resolution, were considered as references. 
The initial test aimed at evaluating the time domain series recorded with these platforms. An impulsive force was applied to the pendulum five seconds after having started the acquisition process. The results of this first test (Figure 2) indicate the good performance of the commercial WSN platforms for measuring high amplitude vibrations. However as expected, for signals with amplitudes below $20 \mathrm{mg}$, the WSN platforms recorded only noise due to the low resolution of the microaccelerometers and ADCs embedded. Note that in this last scenario, it was even possible to observe the digitization lines (see left Figure).
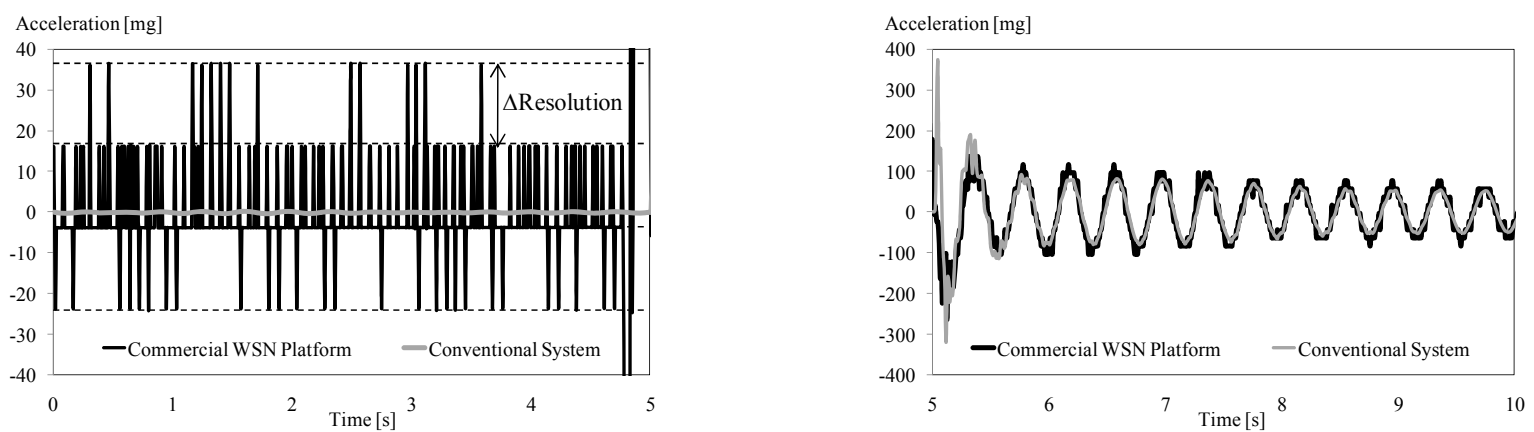

Figure 2 - Time series record of the response of the inverted pendulum under an impulse force test

A second round of evaluation tests was performed to study the accuracy of the wireless systems in tests with longer sampling periods. With this purpose, tests were performed under ambient noise and random impacts. Figure 3 shows the time series recordings and the resultant Welch spectrums, for each of the considered excitation scenarios. The time domain results evidence again the poor performance of the wireless systems for measuring low amplitude vibrations. On the contrary, in random excited tests, the similar response of the conventional and wireless systems is evident. It should be noticed that the results indicate that, due to the lack of implementation of communication protocols in the wireless systems, some data was lost in the recorded time series. For what respects to the frequency domain results, despite the expectable higher noise level of the data recorded with the wireless systems, similar frequency contents were found in both systems in the excited tests. In this scenario, the presence of two peaks, the first one in the band of $0 \mathrm{~Hz}-10 \mathrm{~Hz}$ and the second one close to the band of $30 \mathrm{~Hz}$, were clearly identified. Confirming the poor performance of the commercial WSN platforms in ambient noise tests, in the resultant spectrums no peaks were detected since these showed only noise.
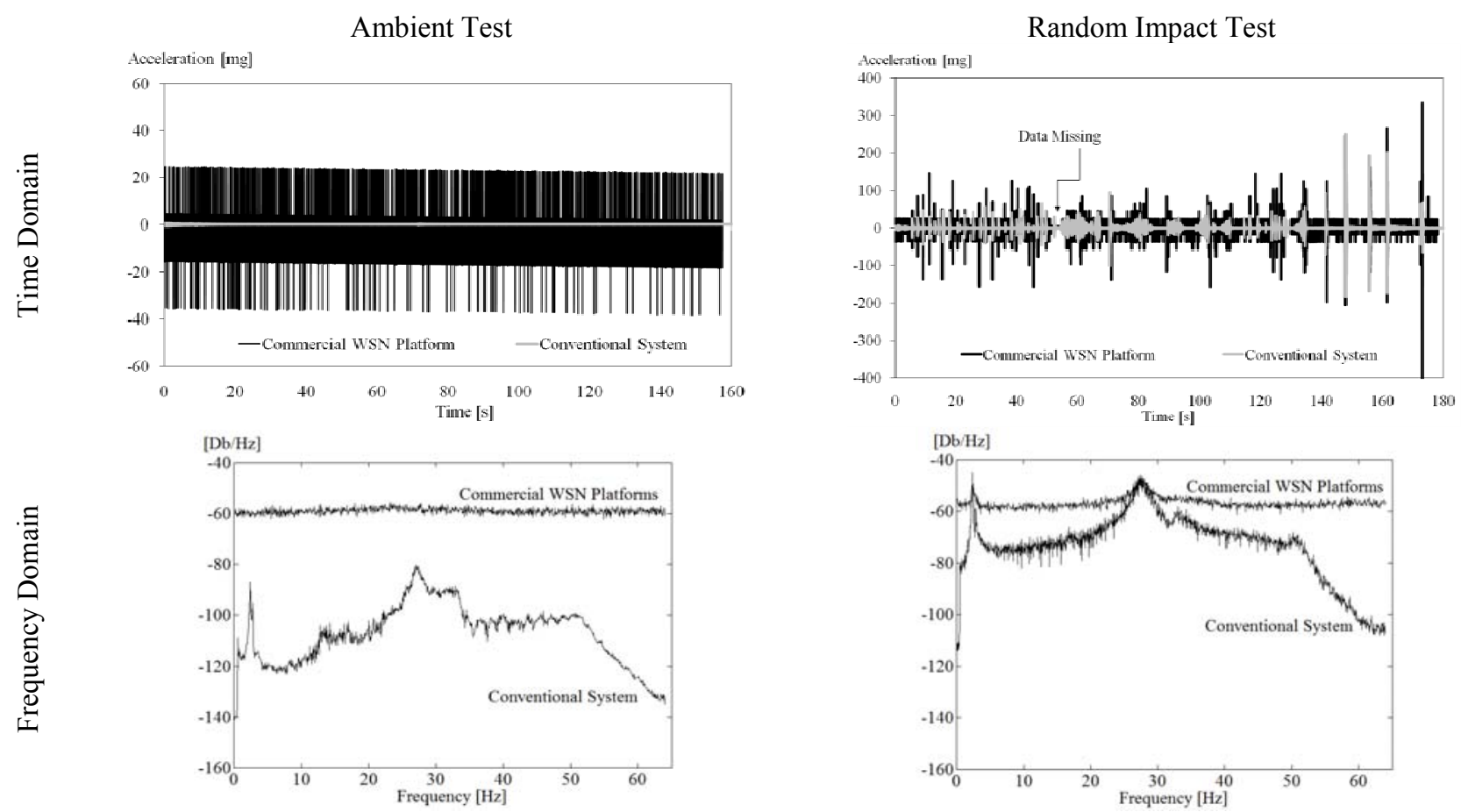

Figure 3 - Time and frequency series results from the data recorded in the inverted pendulum under an impulse force test 
The results of the performed tests showed that the commercial off-the-shelf WSN platforms are not fully suitable for properly identifying the dynamic properties of civil engineering structures. This fact is mainly due to three reasons: 1) low resolution of the embedded microaccelerometers; 2) low resolution of the embedded ADCs; and 3) lack of communication protocols. It should be noticed that the problems related to the energy consumption were not considered since the use of these platforms for long term monitoring was outside the scope of this work.

\section{NEW WIRELESS PLATFORM FOR PERFORMING STRUCTURAL HEALTH MONITORING OF CIVIL ENGINEERING STRUCTURES}

One of the main objectives of the present work was to study the recent progresses on wireless technology and Micro-ElectroMechanical-Systems (MEMS) in order to explore their possible inclusion in the Operational Modal Analysis (OMA) schemes for existent masonry structures. From previous experiences on OMA procedures in this type of structures, a main difficulty was observed related to the low levels of excitation registered in the experimental tests as a consequence of the high stiffness associated to these buildings. Therefore, if OMA tests are planned to be carried out in these structures, the characteristics of the measurement transducers and Data Acquisition (DAQ) equipments should be carefully chosen. From the experience gained on performing OMA in civil engineering structures using conventional and commercial off-the-shelf wireless sensors, the minimum requirements that the new WSN platform must fulfil were defined. According to this, the maximum frequency response range of the system was defined to be from $0 \mathrm{~Hz}$ to $100 \mathrm{~Hz}$ and the maximum sampling frequency to be of $200 \mathrm{~Hz}$. Moreover, the maximum sampling drift must be lower than $5 \mathrm{~ms}$ (1/sampling frequency) and no samples should be lost during the acquisition and communication process. With respect to the measurement sensors, it was decided that triaxial MEMS accelerometers with a maximum measurement range of $\pm 2 \mathrm{~g}$, typical resolution of $1 \mathrm{mg}$, and maximum resolution of $0.1 \mathrm{mg}$ must be included. In the case of the DAQ board, this must include an ADC with at least 16 bits of resolution.

A WSN system can be understood as composed by two parts: the measurement units and the base station (a third part is not considered, the remote connection system, since traditional technology is used for this purpose). The prototype of the WSN platform developed in this work also matches with the mentioned definition following the system architecture presented in Figure 4.

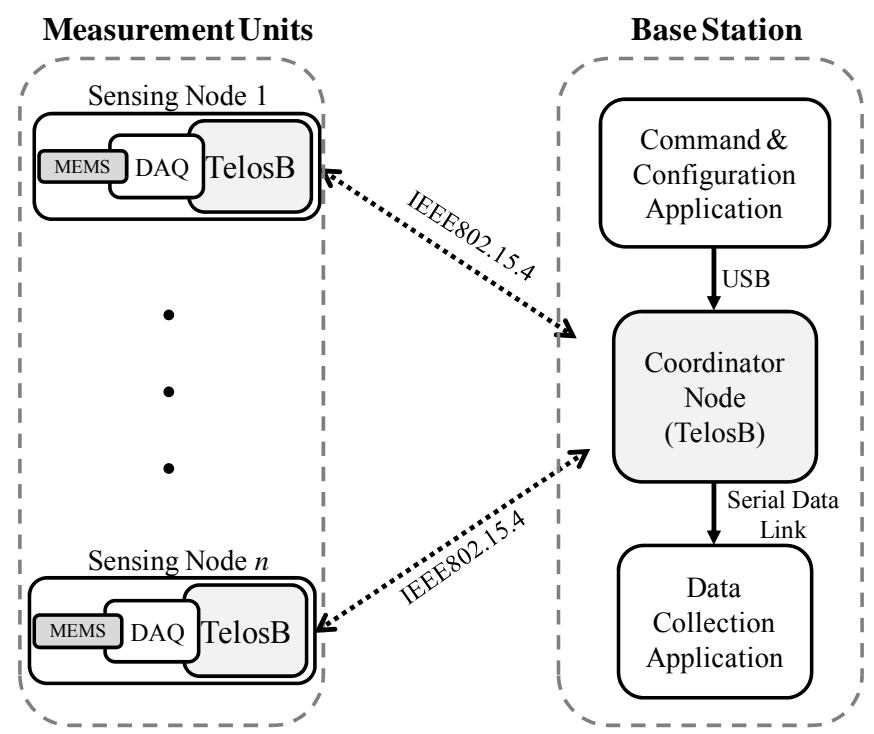

Figure 4 - Overview of the system architecture of the developed WSN platform

In the developed WSN, the wireless communication module in the measurement units and the base station was composed by TelosB platforms [9] working in the radio frequency range of $2.40 / 2.48 \mathrm{GHz}$. These hardware platforms feature an $8 \mathrm{MHz}$ TI MSP430 16-bit microcontroller, a CC2420 RF transceiver (IEEE 802.15.4 compliant), $48 \mathrm{kB}$ of Program memory (insystem reprogrammable flash), $16 \mathrm{kB}$ of EEPROM, and UART communication ports. They also include on-board light, temperature and humidity sensors, whose implementation was outside the scope of this work but; which might be useful in future developments for static SHM applications. The DAQ board was conceived for supporting a high resolution 24 bit $\mathrm{ADC}$ and a $32 \mathrm{MB}$ serial flash memory for storing data samples. Both the ADC and the flash memory are managed by an 8 bits microcontroller (which also handles and pre-formats the acquired ADC samples) and accommodated an analogue 8th order Butterworth filter (to avoid undesired aliasing effects). The energy regulation and management circuitry is supplied 
from sets of four AA size batteries. From the commercial available solutions of MEMS accelerometers; two different types of capacitive triaxial transducers with a measurement range of $\pm 2 \mathrm{~g}$ were selected. The first one was the model ASC 5521-002 (called in this work as MEMS sensor Type A) and the second one the ASC 5631-002 (called as MEMS sensor Type B). The technical specifications' details of these sensors can be found at ASC [3].

With respect to the base station, and as mentioned before, it was composed by one TelosB platform acting as the system's coordinator in addition to the command \& configuration and data collection applications. The role of the coordinator node was to configure and synchronize the whole network using a beacon based solution contemplated in the IEEE 802.15.4 communication protocol. The coordinator node served also as interface between the network of sensors and the command \& configuration application as well as reception point of the forwarded data. The available controls of the command \& configuration application enabled full control over the acquisition configuration parameters (i.e. axis selection, sampling rate, sampling period, etc.) and also provided a quick evaluation of the presence of the system nodes. To complete the data reception process, a VI routine was developed in Labview [14] for data collection purposes. The data is transmitted from the measurement nodes in successive messages containing 8 measurements ( 4 bytes each one) in big-endian format. In this case, the developed application was also in charge of the interpretation and conversion into standard units of the received data as well as their local storage in the central station. More details can be found at [1].

The final appearance of the developed prototype platform is shown in Figure 5. In order to ensure the necessary energy resources in case of possible medium and long term monitoring studies, three sets of four AA batteries where included.

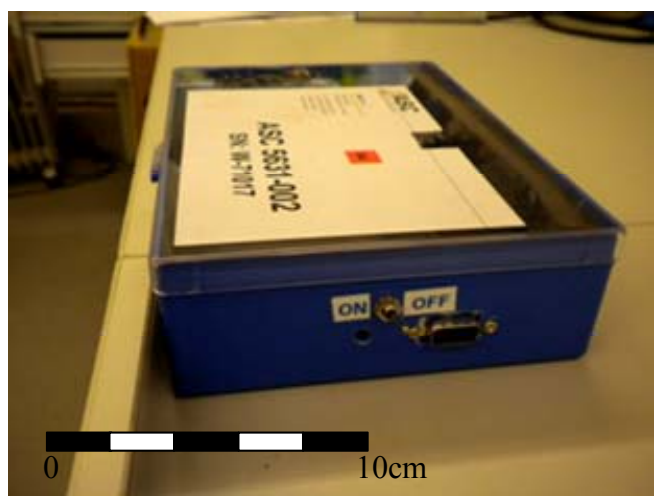

(a)

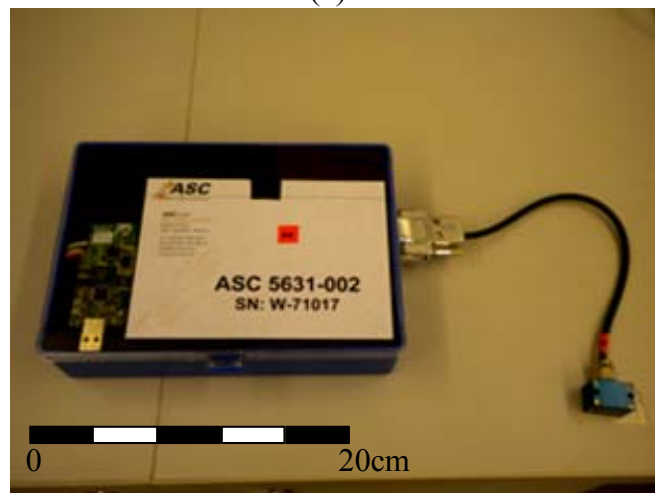

(c)

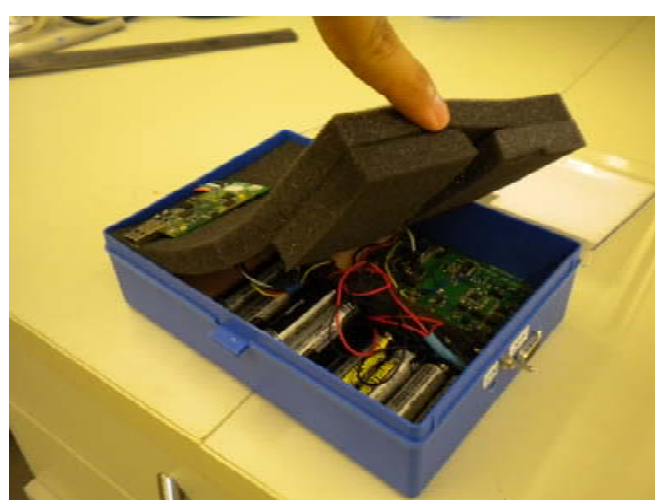

(b)

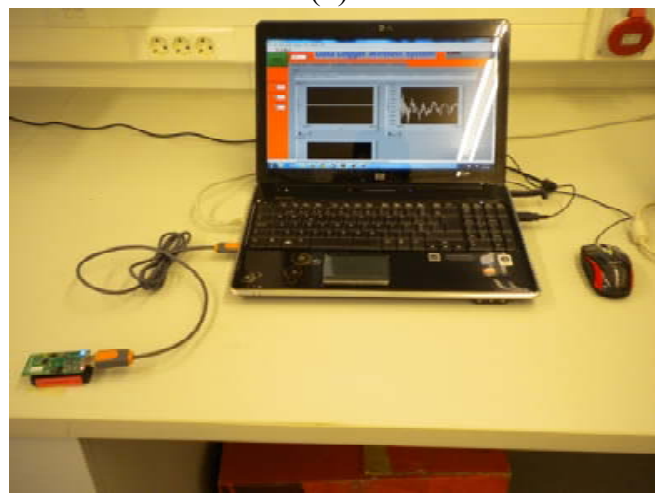

(d)

Figure 5 - Final appearance of the developed WSN solution: (a), (b), (c) measurement unit; and (d) base station

For the laboratory validation tests carried out in this work, the case study of the inverted wooden pendulum previously presented in section 2 was used again. Considering again the conventional wired based systems as reference (high sensitive accelerometers with high resolution ADC), numerous validation tests were carried out. In these experiments, the DAQ stations corresponding to both systems (conventional and wireless) were set for running in parallel aiming at performing measurements at the same instant of time using a common sampling rate of $100 \mathrm{~Hz}$.

The initial time domain evaluation tests were carried out considering one measurement point at the top of the pendulum. As shown in Figure 6, one of each measurement units was located at that point (MEMS Accelerometers Type A and B as well as the conventional wired based transducers). 


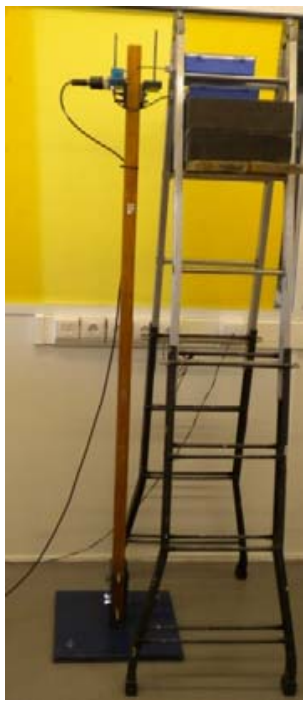

(a)

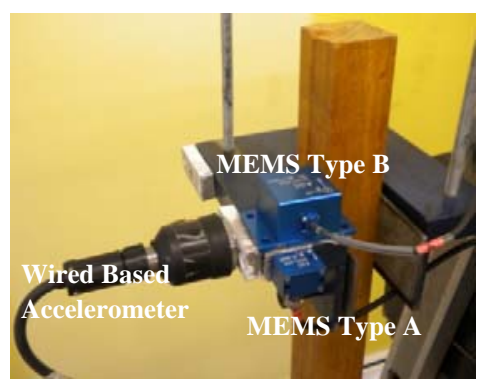

(b)

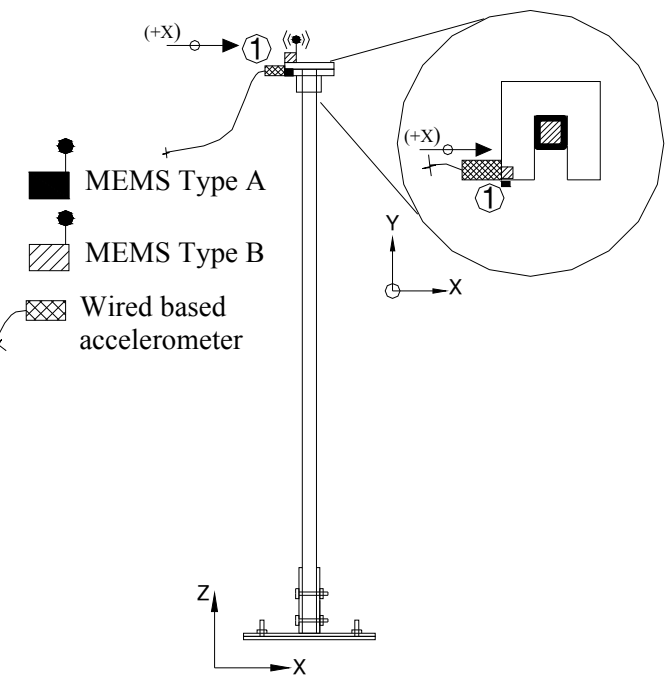

(c)

Figure 6 - Description of the arrangement of sensors for the time domain evaluation tests. (a) Inverted pendulum in the laboratory; (b) close up of the wired and wireless based sensors arrangement; and (c) scheme of the sensors deployment

The performance of the developed platforms in high and low amplitude excitations was evaluated when an impulse force was applied to the pendulum. For this test, a short sampling period of 10 seconds was considered. The time series recordings of the performed test are presented in Figure 7. The results indicated that for moderate amplitude vibrations (above $1 \mathrm{mg}$ ) the recordings of the three systems are very similar. However, as shown in the bottom left figure, for signals with amplitudes below this limit, the solution corresponding to the MEMS Accelerometer Type A has limitations. On the contrary, the system with MEMS Accelerometer Type B presents outstanding results maintaining a remarkable similitude with the conventional transducers, even in low noise environments. It is important to state that, even in the case of the MEMS Type A, the considered solutions had at least 20 times better performance in comparison to the commercial WSN solution presented in the previous section.
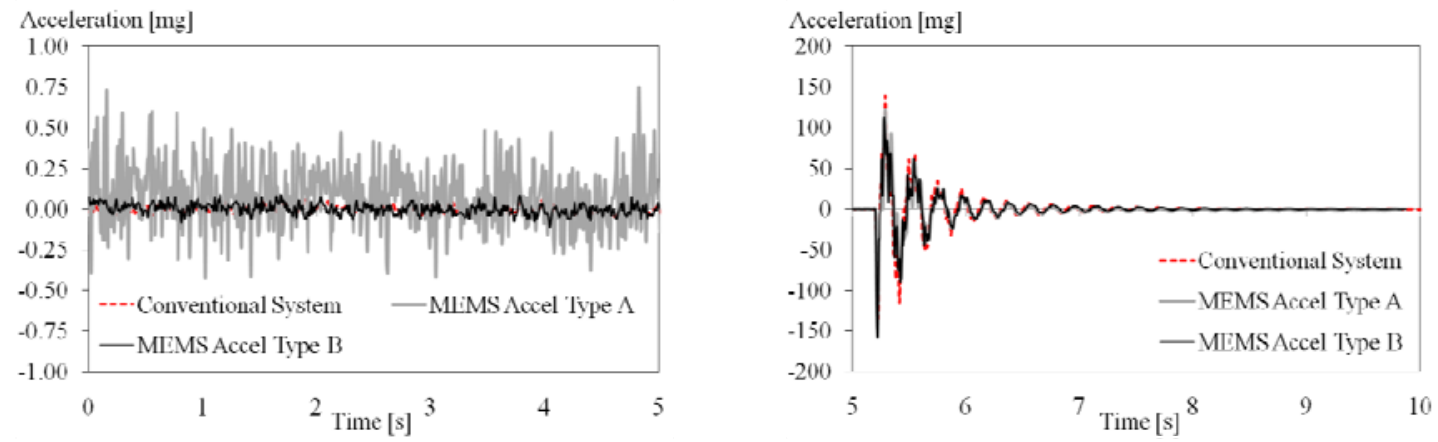

Figure 7 - Time series recordings of the response of the inverted pendulum under an impact force test - Validation Tests for New WSN Platforms

Next, the performance of the developed solution was evaluated considering tests with longer sample periods. Using the same deployment of sensors and 60 seconds for the sampling time, two rounds of tests were carried out: one considering light random impact excitations and the other ambient noise. Note that even if longer sampling periods of $1000-2000$ times the structure's first period are recommended for practical recommendations [19], shorter measurement times were considered due to time consuming data transmission process in this first prototype system. In this paper only the results of the solution using the MEMS accelerometers Type B will be presented since better performance in low noise environments was obtained. Figure 8 shows the time series recordings of the studied systems in each of the considered noise scenarios. The results showed the good performance of the developed system for registering low amplitude time domain events since vibrations with amplitudes above $0.1 \mathrm{mg}$ were accurate acquired. For what respects to the frequency domain, the resultant spectrum of the wired and wireless based systems presented outstandingly similitude. 

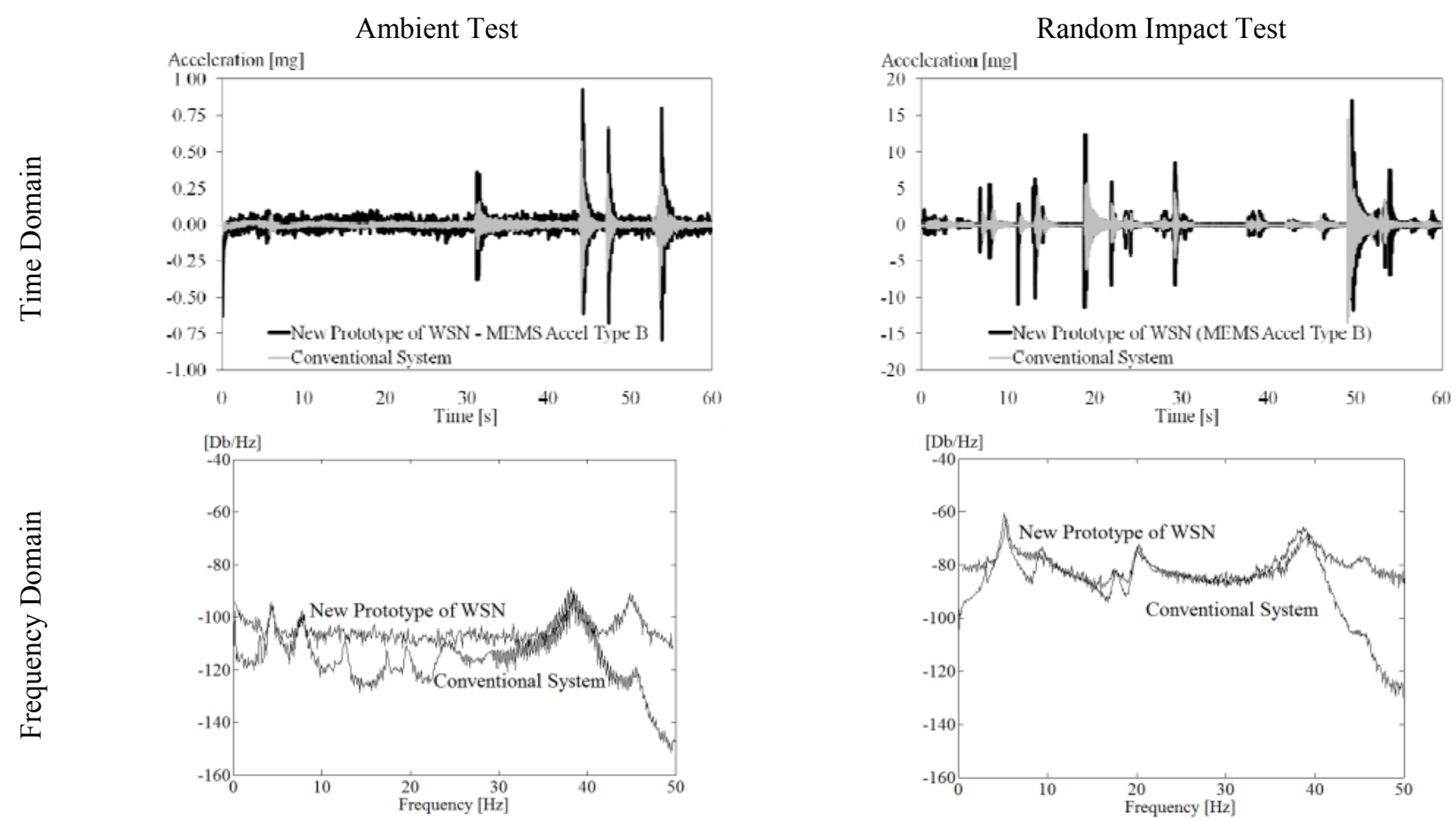

Figure 8 - Time and frequency series results from the data recorded in the inverted pendulum under an impulse force test

The modal validation tests considered the location of three measurement nodes at the top of the pendulum. In this case, the solution with MEMS accelerometers type A was considered since only one MEMS type B was available. As done before, the response of the pendulum was studied considering 60 seconds of sampling time and two excitation scenarios (moderate and low noise environments). In the collected time domain series acceleration peaks of $3.93 \mathrm{mg}$ and $0.35 \mathrm{mg}$ were registered, which corresponded to signals with RMS values of $0.23 \mathrm{mg}$ and $0.03 \mathrm{mg}$ for the medium and low noise environments, respectively.

For the modal identification process, it was considered that the information of interest is related only to the estimation of the first three structural mode shapes (translation in the two principal axes and rotation). Since the results of the developed platforms are imprecise in low noise environments, Figure 9 presents the results of the identified modal shapes only for the case of medium noise environments. The results of the medium excited environments evidenced the high accuracy of the measurements of the developed system. As it is possible to observe, the first two modes of the structure were identified with no uncertainties (MAC values close to 1) while for the case of the third mode, a slight difference with the results of the conventional systems was registered.

- - MEMS Type A

- Conv. System

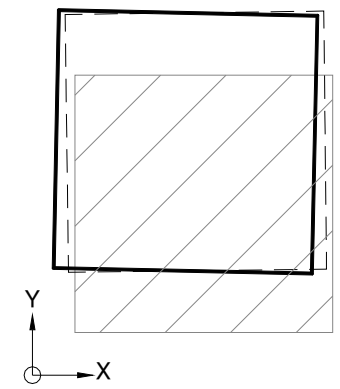

MAC 1-1: 0.90

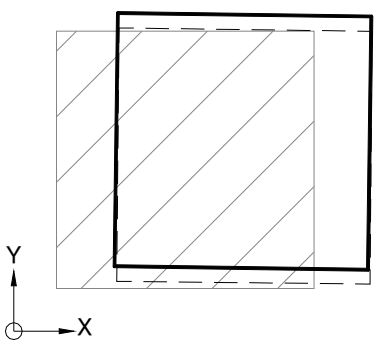

MAC 2-2 : 0.92

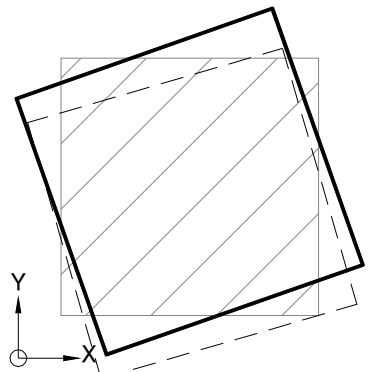

MAC 3-3 : 0.65

Figure 9 - First three mode shapes estimated from the experimental modal analysis tests in the pendulum first configuration Validation Tests for New WSN Platforms 


\section{CONCLUSIONS}

The present work explores the possible inclusion of Wireless Sensor Networks (WSN) in the Operational Modal Analysis (OMA) of civil engineering structures. With this purpose, the possibilities of the commercial off-the-shelf solutions on WSN platforms were first evaluated in detail. Several tests were carried out using these platforms and the results indicate that this technology, as it is commercialized, has no direct application in this type of studies. The main reasons are the low resolution of the accelerometers and the ADCs embedded and the lack of communication protocols that assure not only a proper synchronization among nodes but also reliability in the communication processes.

Once the limitations of the ready-to-use WSN solutions were identified, a joint team involving electronic and communication engineers developed a prototype WSN system aiming at fulfilling the demanding requirements of OMA tests in civil engineering structures. The laboratory and field validation tests carried out using the developed system indicate that this can be positively considered as alternative to the conventional wired based systems in monitoring works where vibrations with amplitudes over $0.10 \mathrm{mg}$ are expected. In these scenarios, the results of the modal analysis process, by means of the detected frequencies and mode shapes evidenced outstanding accuracy comparable to the conventional wired based systems.

\section{ACKNOWLEDGMENTS}

The authors gratefully acknowledge Alban, European Union Programme of High Level Scholarships for Latin America, for the financial support of the PhD studies of the first author with the scholarship number E07D400374PE.

\section{REFERENCES}

[1] Aguilar, R. Dynamic Structural Identification using Wireless Sensor Networks. PhD Thesis, University of Minho, Guimaraes, (2010), Available at www.civil.uminho.pt/masonry

[2] Aoki, S.; Fujino, Y.; and Abe, M. Intelligent Bridge Maintenance System Using MEMS and Network Technology. Smart Systems and NDE for Civil Infrastructures - SPIE, San Diego, CA, Vol. 5057, (2003), pp. 37-42.

[3] ASC. Advanced Sensors Calibration. www.asc-sensors.de. Accessed December, (2009).

[4] Baptista, M.A.; Mendes, P.; Afilhado, A.; Agostinho, L.; Lagomarsino, A.; and Victor, L.M. Ambient Vibration Testing at N. Sra. do Carmo Church, Preliminary Results. Proc. of the 4th International Seminar on Structural analysis of Historical Constructions, Padova, Italy, (2004), pp. 483-488.

[5] Casarin, F.; and Modena, C. Dynamic Identification of S. Maria Assunta Cathedral, Reggio Emilia, Italy. Proc. of the 2nd International Operational Modal Analysis Conference, IOMAC, Copenhagen, Denmark, (2007), pp. 637-644.

[6] Ceriotti, M.; Mottola, L.; Picco, G.P.; Murphy, A.L.; Guna, S.; Corra, M.; Pozzi, M.; Zonta, D.; and Zanon, P. Monitoring Heritage Buildings with Wireless Sensor Networks: The Torre Aquila Deployment. International Conference on Information Processing in Sensor Networks, IPSN09, San Francisco, USA, (2009).

[7] Chung, H.C.; Enomoto, T.; Shinozouka, M.; Chou, P.; Park, C.; Yokoi, L.; and Morishita, S. Real-time Visualization of Structural Response with Wireless MEMS Sensors. 13th World Conference on Earthquake Engineering, Vancouver, BC, (2004).

[8] Costa, C.; Arêde, A.; and Costa, A. Caracterização Dinâmica e Análise da Ponte da Lagocinha. Proc. of the 6to Congresso de Sismologia e Engenharia Sísmica, Guimarães, Portugal, (2004), pp. 931-942.

[9] Crossbow. Crossbow Wireless Sensors Network. www.xbow.com. Accessed December, (2009).

[10] Farrar, C.R.; and Worden, K. An Introduction to Structural Health Monitoring. Philosophical Transactions of the Royal Society, 365, (2007) 303-315.

[11] Gentile, C.; and Saisi, A. Dynamic-based F.E. Model Updating to Evaluate Damage in Masonry Towers. Proc. of the 4th International Seminar on Structural analysis of Historical Constructions, Padova, Italy, (2004), pp. 439-449.

[12] Ivorra, S.; and Pallarés, F.J. A Masonry Bell-Tower Assessment by Modal Testing. Proc. of the 2nd International Operational Modal Analysis Conference, IOMAC, Copenhagen, Denmark, (2007), pp. 269-276. 
[13] Jaishi, B.; Ren, W.X.; Zong, Z.H.; and Maskey, P.N. Dynamic and Seismic Performance of Old Multi-Tiered Temples in Nepal. Engineering Structures, 25, (2003) 1827-1839.

[14] Labview. LabVIEW User Manual, Release 8.0. National Instruments Corporation, USA, 2006.

[15] Lynch, J.P.; Sundararajan, A.; Law, K.H.; Kiremijdian, A.S.; Carryer, E.; Sohn, H.; and Farrar, C.R. Field Validation of a Wireless Structural Health Monitoring System on the Alamosa Canyon Bridge. Smart Structures and Materials: Smart Systems and Nondestructive Evaluation for Civil Infrastructures - SPIE San Diego, CA, Vol. 5057 (2003), pp. $267-278$.

[16] Lynch, J.P.; Wang, Y.; Law, K.H.; Yi, J.-H.; Lee, C.G.; and Yun, C.B. Validation of a Large-scale Wireless Structural Monitoring System on the Geumdang Bridge. Proc. of the International Conference on Safety and Structural Reliability (ICOSSAR), Rome, Italy, (2005).

[17] Ou, J.P.; Li, H.; Xiao, Y.Q.; and Li, Q.S. Health Dynamic Measurement of Tall Building Using Wireless Sensor Network. Proc. of the Smart Structures and Materials, SPIE, San Diego, CA, USA, (2005), pp. 205-215.

[18] Pakzad, S.N.; Fenves, G.L.; Kim, S.; and Culler, D. Design and Implementation of Scalable Wireless Sensor Network for Structural Monitoring. Journal of Infrastructure Systems, 14(1), (2008) 89-101.

[19] Ramos, L.F. Damage Identification on Masonry Structures Based on Vibration Signatures. PhD Thesis, University of Minho, Guimaraes, Portugal, (2007), Available at www.civil.uminho.pt/masonry

[20] Ramos, L.F.; Alaboz, M.; Aguilar, R.; and Lourenço, P.B. Dynamic Identification and FE Updating of S. Torcato Church, Portugal. 28th International Modal Analysis Conference, IMAC. SEM, Jacksonville, Florida, USA, (2010).

[21] Ramos, L.F.; Casarin, F.; Algeri, C.; Lourenço, P.B.; and Modena, C. Investigations Techniques Carried out on the Qutub Minar, New Delhi, India. Proc. of the 5th International Conference of Structural Analysis of Historical Constructions, New Delhi, (2006), pp. $633-640$.

[22] Rebelo, C.; Júlio, E.; and Costa, D. Modal Identification of the Coimbra University Tower. Proc. of the 2nd International Operational Modal Analysis Conference, Copenhagen, Denmark, (2007), pp. 177-184.

[23] Schmidt, T. Dynamic Behaviour of Twin Bell Towers. Proc. of the 2nd International Operational Modal Analysis Conference, Copenhagen, Denmark, (2007), pp. 261-268.

[24] Straser, E.G.; and Kiremijdian, A.S. A Modular, Wireless Damage Monitoring System for Structures, The John A. Blume Earthquake Engineering Center, Report No.128, (1998).

[25] Weng, J.-H.; Loh, C.-H.; Lynch, J.; Lu, K.-C.; Lin, P.-Y.; and Wang, Y. Output-Only Modal Identification of a CableStayed Bridge using Wireless Monitoring Systems. Engineering Structures, 30(7), (2008) 1820-1830. 